\title{
Modelo matemático para determinar la correlación entre parámetros fisicoquímicos y la calidad sensorial de café Geisha y Pacamara de Panamá
}

Aracelly Vega1, Javier A. De León' ${ }^{1}$, Stephany M. Reyes ${ }^{1}$ y José M. Gallardo²*

(1) Centro de Investigación en Recursos Naturales, Universidad Autónoma de Chiriquí. Apartado Postal 0427, Chiriquí, República de Panamá. (correo-e: aravega@cwpanama.net, javier.deleon@unachi.ac.pa, stephany.reyes@unachi.ac.pa).

(2) Centro Regional Universitario de Chiriquí, David, Universidad Tecnológica de Panamá, República de Panamá. (correo-e: jose.gallardo@utp.ac.pa)

${ }^{*}$ Autor a quien debe ser dirigida la correspondencia.

Recibido Jul. 2, 2020; Aceptado Ago. 26, 2020; Versión final Oct. 14, 2020, Publicado Feb. 2021

Resumen

El objetivo de esta investigación fue desarrollar un modelo matemático que permita predecir el puntaje que indica la calidad sensorial del café. Se utilizaron 11 parámetros fisicoquímicos de 34 muestras de café y el puntaje del panel de 12 jueces de catación. Se realizó un análisis de regresión múltiple para determinar la relación entre los parámetros fisicoquímicos y la calidad sensorial. Se obtuvo el cuadrado del coeficiente de correlación ajustado, los coeficientes de cada parámetro dentro del modelo y los valores $p$ de cada uno de ellos. Los resultados muestran que los parámetros ácido 4-cafeoilquínico(4-CQA), pH, color, densidad y acidez, tienen una influencia negativa sobre la calidad sensorial, mientras que los parámetros, ácido 3 cafeoilquínico(3-CQA), ácido 5-cafeoilquínico(5-CQA), humedad, Brix, y cafeína tienen una influencia positiva. Los parámetros 3-CQA, 4-CQA y pH mostraron los menores valores $\mathrm{p}$. Se concluye que el modelo permite estimar el rango en el cual se puede encontrar el puntaje de un lote de café.

\section{Mathematical model to determine the correlation between physicochemical parameters and the sensory quality of Panama Geisha and Pacamara coffee}

\begin{abstract}
The main objective of this research study was to develop a mathematical model to predict the score that indicates coffee's sensory quality. To develop the model, the study examined 11 physicochemical parameters, 34 coffee samples, and the judging scores from a 12 cupping judge panel. A multiple regression analysis was performed to determine the relationship between physicochemical parameters and sensory quality to obtain the square of the adjusted correlation coefficient, the coefficients for each parameter within the model, and their p-values. The results show that the parameters 4-caffeoylquinic acid (4-CQA), pH, color, density, and acidity, have a negative influence on sensory quality. The parameters 3-caffeoylquinic acid (3-CQA), acid 5-caffeoilquinic (5-CQA), humidity, Brix, and caffeine have a positive influence on sensory quality. In addition, the parameters 3-CQA, 4-CQA, and pH showed the lowest $\mathrm{p}$ values. It is concluded that the model developed here allows estimating the proper range required to score a coffee batch.
\end{abstract}

Keywords: geisha coffee; pacamara; matemathical model; cup quality; sensory 


\section{INTRODUCCIÓN}

El café es uno de los productos más importantes del comercio internacional y para su aceptabilidad se establecen diferentes criterios de calidad. El olor y el sabor de la bebida de café son los criterios más importantes para la evaluación de la calidad del mismo y una de las principales motivaciones para la preferencia por los consumidores. La calidad del café depende del genotipo, de las condiciones ambientales, de factores propios del sistema de producción, del aspecto físico, de la composición química de los granos verdes o tostados y de las características de la bebida final. El olor y el sabor de una bebida de café está relacionada con la composición fisicoquímica del grano, del proceso de cosecha, tostado, almacenamiento y de la forma de preparar la bebida (Pérez-Hernández et al., 2012; Cheng et al., 2016; Laukalēja y Krūma, 2018; Toci \& Boldrin, 2018).

Estudios han demostrado que hay una correlación entre la calidad de la taza basada en atributos sensoriales y los componentes químicos del café (Farah et al., 2006; Ribeiro et al., 2011; Bressanello et al., 2017; Barbosa et al., 2019). Se ha encontrado que atributos sensoriales del café tales como acidez, cuerpo, dulzura, amargura, aroma y otros están relacionados con componentes químicos del grano de café verde y tostado. Según Barbosa et al., (2019), en un estudio de café arábica verde utilizando espectroscopía NIR encontró que la cafeína, proteína, ácidos clorogénicos y acidez bajan la CT de la taza y sacarosa/acidez, cafestol/kahweol suben la CT de la taza. Este perfil de la composición del café verde puede ser un indicativo de la calidad sensorial de la bebida de café, información relevante para productores e industriales ya que el grano de café verde es el material usado para el comercio del café.

Se han generado modelos matemáticos para predecir las propiedades físicas y organolépticas de la bebida de café utilizando diversas herramientas químicas como espectroscopía de resonancia magnética nuclear RMN (Wei et al., 2014), espectroscopía de infrarrojo cercano (Ribeiro et al., 2011), imágenes hiperespectrales (HSI) (Caporaso et al., 2018). Ribeiro et al.(2011), en un estudio del café arábica tostado utilizando espectroscopía NIR encontró que los lípidos y proteína están relacionados al atributo de cuerpo, cafeína y ácidos clorogénicos al atributo dulzura, ácidos clorogénicos a acidez y sabor, Cafeína, trigonela, ácidos clorogénicos, polisacáridos, sacarosa y proteína relacionados al atributo limpieza y CT. También se han desarrollado modelos matemáticos predictivos para determinar propiedades físicas del café durante la etapa del tostado (Bustos-Vanegas et al., 2017).

Actualmente las propiedades sensoriales del café son evaluadas por un panel de jueces entrenados y seleccionados, utilizando un método de catación y con el cual se genera un puntaje que indica la calidad del café. A pesar de que este método es fiable, es muy costoso, subjetivo y poco rápido por lo que la industria del café está interesada en encontrar métodos analíticos más objetivos, simples y rápidos para evaluar la calidad de la bebida del café con propósitos comerciales (Tolessa et al., 2015). En Panamá se ha desarrollado la industria de los cafés especiales cuya calidad se evalúa por el método de la "taza". Para esta evaluación, se realiza cada año un evento de catación nacional siguiendo los protocolos desarrollados y estandarizados por la industria del café de especialidad a nivel global.

El protocolo base es el desarrollado por la Specialty Coffee Association of America (SCAA) (SCAA, 2015). Este método está basado en el análisis sensorial, descriptivo y cuantitativo de la bebida, análisis que es ejecutado por un grupo de jueces entrenados y seleccionados. En la catación, los granos de café son evaluados, tomando en cuenta los atributos principales que componen el perfil sensorial del café, que son: fragancia/aroma, acidez, cuerpo, sabor, resabio, uniformidad, limpieza, dulzura, balance, atributos totales y a cada atributo se le califica en una escala de 6 a 10 puntos. La suma de las calificaciones individuales de todos los atributos constituye el puntaje final, el cual representa la calidad del café (Toci y Boldrin, 2018).

Los lotes de café (Coffea arabica var. geisha) con las mejores puntuaciones de este panel actualmente se comercializan en el mercado por precios de hasta los 2200 dólares por kg., adquiridos por clientes de países como Japón, China, Estados unidos y Australia, entre otros, quienes lo prefieren por su aroma y sabor. Según Vega et al., $(2014,2017,2018)$, el café geisha proporciona el mayor contenido de ácidos clorogénicos por taza de café, menor contenido de cafeína, alta actividad antioxidante y presenta una relación ácidos clorogénicos/cafeína (CGAs/Caf) en el rango de 2.48 a 3.85, por lo que se puede proponer como fuente de antioxidantes de uso en varias industrias como la alimentaria, farmacéutica y nutracéutica.

El propósito del presente estudio fue desarrollar un modelo matemático que permita predecir el puntaje como un indicativo de las propiedades organolépticas de muestras desconocidas de café, usando los valores de las propiedades fisicoquímicas obtenidas en análisis de laboratorio junto con la calificación del panel de jueces de la catación nacional de cafés especiales de Panamá. Que este modelo, además de predecir el puntaje, sirva como herramienta de apoyo a los jueces en el proceso de catación nacional y a los productores para tener más conocimiento sobre las características de su producto. 


\section{METODOLOGÍA}

La metodología se presenta en varias subsecciones las cuales son: Proceso de catación, obtención de las muestras, estándares, reactivos y solventes, análisis fisicoquímico de la bebida de café y calibración del modelo multivariable.

\section{Proceso de catación}

La catación o evaluación sensorial se realizó siguiendo protocolos desarrollados y estandarizados por la industria del café de especialidad a nivel global. Se aplicó el protocolo desarrollado por la Specialty Coffee Association (antes SCAA) para evaluar la calidad sensorial en base a un puntaje que para cafés especiales el puntaje debe ser al menos superior a 80 . Entre 80 y 85 la muestra se considera de muy buena calidad, entre 85 y 90 es de excelente calidad y de 90 a 100 es de calidad excepcional. El puntaje final es la suma de los puntajes individuales de las 10 características que se evaluaron. Los lotes se identificaron con los números que fueron colocados al momento de recibirse el café sin tostar en la bodega de almacenaje controlada por los auditores externos. El tueste del café fue realizado en un solo equipo de tostación y el control del color de tueste se realizó midiendo el mismo tostado en grano y luego el mismo café en molido. De acuerdo a las pruebas de taza se definió el nivel agtron más o menos un número de varianza (Ejm. Agtron $64 \pm 3$ en grano y $66 \pm 3$ en molido). Para la escogencia de los niveles de agtron, para la competencia, se realizaron pruebas de taza (o catación a ciegas) para definir los parámetros de acuerdo al comportamiento de la cosecha del café. Igualmente se realizó catación de café para determinar el grado de molido para la extracción deseada. Se utilizaron cuatro molinos graduados para realizar la molienda.

Por cada café participante, se evaluó entre cuarenta y cuarenta y ocho tazas de café, en grupos de cinco o seis tazas por mesa, en un mínimo de ocho mesas. A cada mesa se le asignó uno o dos jueces, y se cataron hasta diez cafés, por mesa, por ronda. De cada muestra se pesaron $13 \mathrm{~g}$ de café tostado en grano, para cada taza de café a catar. Para la preparación de la bebida, se le agregaron $140 \mathrm{~g}$ de agua a $96^{\circ} \mathrm{C}$ a $13 \mathrm{~g}$ de café molido. Para el proceso de calibración se evaluaron dos muestras utilizando el mismo procedimiento de evaluación estandarizada de la SCAA, generando una puntuación final para ambas muestras. Al terminar, todos los jueces dieron su puntaje para cada muestra y se verificó el nivel de dispersión, que debe ser el mínimo. Cuando se llegó a un acuerdo de puntaje, se inició el proceso de catación de los lotes a concurso.

Cuando se inició la ronda de cata, las mesas se prepararon con el café molido, se evaluó primero la fragancia: café molido en seco; después se procedió a agregar agua caliente al café molido. A los 3 minutos de haber vertido el agua, se "rompe" la capa, formada por el café molido húmedo y se evalúo el aroma del café. Cada juez ejecutó el mismo movimiento, para todas las tazas a probar, obteniendo una extracción lo más uniforme posible, entre las muestras de café, cuando se evaluó el aroma. Al finalizar el proceso de rompimiento de capa y evaluación del aroma, se procedió a la limpieza de la superficie del líquido o bebida obtenida.

Este procedimiento elimina los materiales sólidos flotantes al igual que la espuma para que se pueda probar el líquido a través de una aspiración. Esta aspiración particular permite que el líquido se distribuya por toda la cavidad bucal apoyando la evaluación de la experiencia gustativa en función de los demás parámetros a medir. Al finalizar la ronda, se obtuvo un puntaje como resultado final para cada muestra evaluada. Estos resultados se enviaron al centro de proceso en donde se consolidó en un puntaje final, en este caso, a través del cálculo de la mediana.

\section{Obtención de la Muestra}

Las muestras de café que se usaron en este estudio fueron de café con proceso natural y café con proceso lavado. El café con proceso natural se obtuvo mediante el método de secar la cereza entera después de colectada. Estas cerezas se secaron al sol y luego pasaron al proceso de desmucilaginación y tostado. El café con proceso lavado se obtuvo quitando la pulpa de café ya sea usando una máquina desmucilaginadora o por fermentación húmeda o seca y el café sin pulpa se sometió a un proceso de lavado y secado para luego tostarlo. Para el estudio se obtuvieron 10 muestras (30 réplicas) de bebida de café Geisha con proceso natural (código D), 10 muestras (30 réplicas) de bebida de café Geisha con proceso lavado (código $\mathrm{C}$ ), 7 muestras (21 réplicas) de bebida de café Pacamara con proceso natural (código E) y 7 muestras (21réplicas) de bebida de café Pacamara con proceso lavado (código E). Todas las muestras tienen aproximadamente $12 \%$ de humedad antes del tostado, el color del tostado es de 63-69 agtron y densidad promedio de $70 \mathrm{~kg} /$ hectolitro.

Durante el proceso de catación se prepararon las bebidas. Cada bebida fue preparada con 13 gramos de café molido a los cuales se les agregó $140 \mathrm{~g}$ de agua a $96^{\circ} \mathrm{C}$, para cada taza. De cada muestra codificada 
se colectaron 3 réplicas. Se escogieron al azar 3 mesas y de cada una se preparó una muestra con el contenido de 3 tazas de la misma mesa, para obtener $100 \mathrm{~mL}$ de muestra. Las muestras fueron traídas al laboratorio en envase ámbar de plástico y vidrio, los cuales fueron almacenados en nevera a una temperatura entre $10-15^{\circ} \mathrm{C}$, hasta realizar el análisis.

\section{Estándares, reactivos y solventes}

Los estándares de ácido 5-cafeoilquínico (pureza: $\geq 95.0 \%$ ), ácido 3-cafeoilquínico (pureza: $\geq 98.0 \%$ ), fueron comprados de Sigma-Aldrich (St. Louis, MO). La cafeína (pureza: $\geq 98.5 \%$ ) fue obtenida de Spectrum Quality Products (New Brunswick, NJ). Los solventes metanol (pureza: 99.8\%) y ácido trifluoroacético (pureza: 99.5\%) de grado HPLC obtenidos de Tedia (United States).

Análisis fisicoquímico de la bebida de café

Los análisis fisicoquímicos realizados a la bebida de café fueron: acidez total, $\mathrm{pH}$, grados brix, densidad, proteína y compuestos bioactivos como cafeína, ácido 3-cafeoilquínico, 4-cafeoilquínico y 5-cafeoilquínico.

\section{Determinación de acidez total}

Se utilizó una alícuota de $10 \mathrm{~mL}$ de la bebida de café y se pasó a un matraz Erlenmeyer de $250 \mathrm{~mL}$, se adicionó $100 \mathrm{~mL}$ de agua destilada y 6 gotas de indicador de fenolftaleína. Posteriormente, se tituló con $\mathrm{NaOH}$ 0.1 M hasta cambio de color según el método AOAC 920.92 (AOAC, 2010).

\section{$\mathrm{pH}$ de muestras}

El pH se midió colocando directamente el electrodo del potenciómetro (OAKTON serie pH 1100, Illinois, EE.UU), sobre la bebida de café a temperatura ambiente de acuerdo al método AOAC 970.21(AOAC, 2010).

\section{Determinación de Brix}

Una gota de la bebida de café se colocó en el refractómetro (AGON 0-32\%, Thermo Fisher Scientific, EE.UU), a una temperatura de $20^{\circ} \mathrm{C}$. La lectura se realizó por triplicado. El resultado fue expresado como \% sólidos solubles según el método AOAC 932.14C (AOAC, 2010).

\section{Determinación de densidad}

Se utilizaron volumétricos de $10 \mathrm{~mL}$ lavados y secos, se colocó en una balanza analítica (AA-250, Denver Instrument Company), y se adicionó con un gotero la muestra de café hasta la marca de aforo y se registró el peso.

\section{Determinación de proteína}

Se pesaron 5 gramos de la bebida de café y se determinó por el método Kjeldahl, AOAC 2001.11. Se obtiene el \% de nitrógeno y se multiplica por el factor de 6,25 para obtener el \% de proteína. (AOAC, 2010).

\section{Identificación y cuantificación de compuestos bioactivos por HPLC}

La bebida de café se diluyó en una proporción de $1 \mathrm{~mL}$ de café en $9 \mathrm{~mL}$ de agua y antes de inyectar en el equipo se pasó a través de filtros de jeringa de $0.45 \mu \mathrm{m}$ y diámetro de $25 \mathrm{~mm}$. Se usó la metodología propuesta por Wen et al., (2005), con algunas modificaciones. Se utilizó un sistema HPLC Agilent 1260 Infinity (California, EE.UU), con bomba cuaternaria (G1311C), compartimiento de columna termostatizado (G1316A), detector de arreglo de diodos (G1315D) e inyección manual (Rheodyne). La separación se llevó a cabo con una columna ZORBAX SB-C18 Stable Bond Analytical $(4.6 \times 150 \mathrm{~mm}, 5 \mu \mathrm{m})$. Las condiciones de separación establecidas fueron las siguientes: volumen de inyección de $20 \mu \mathrm{L}$, flujo de $0.4 \mathrm{~mL} / \mathrm{min}$, fase móvil A (agua con $0.02 \%$ de ácido trifluoroacético), fase móvil B (metanol con 0.02\% de ácido trifluoroacético), temperatura de $25^{\circ} \mathrm{C}$ y tiempo de corrida de $60 \mathrm{~min}$. El gradiente programado fue el siguiente: 98:2 (solvente A: solvente B) a los $0 \mathrm{~min}, 68: 32$ a los $30 \mathrm{~min}, 60: 40$ a los $40 \mathrm{~min}, 60: 40$ a los 45 min y $98: 2$ a los 50 min y longitud de onda de 325 y $280 \mathrm{~nm}$.

La identificación se llevó a cabo por la comparación del tiempo de retención de la muestra con el tiempo de retención de los respectivos estándares. La determinación de la concentración de los compuestos se realizó utilizando curvas de calibración hechas para cada estándar, ácido 5-CQA y 3-CQA en un rango de 0.5 a 100 mg/L, y la cafeína se cuantificó en un rango de 10 a 250 mg/L. Para cuantificar el ácido 4-CQA, se utilizó directamente la curva del ácido 5-CQA, por la similitud del coeficiente de extinción molar de estos isómeros (Naegele, E., 2016). 


\section{Calibración de modelo multivariable}

Se realizó un análisis de regresión múltiple en Microsoft Excel (Office 365) para determinar la relación entre los once parámetros fisicoquímicos (variables independientes) y la calidad sensorial, la cual es calculada como la mediana del puntaje de los jueces (variable dependiente). De los resultados del análisis se obtuvo el cuadrado del coeficiente de correlación ajustado $\left(R_{\text {ajustado }}{ }^{2}\right)$, los coeficientes de cada parámetro dentro del modelo obtenido mediante la regresión lineal múltiple $\left(b_{i}\right)$ y los valores $p$ de cada uno de los once parámetros (valor-p). Valores p pequeños indican baja probabilidad de que la hipótesis de nulidad sea cero, por lo que los parámetros cuya $p$ es más baja se considera que tienen la mayor probabilidad de ser significativos para el modelo.

\section{RESULTADOS Y DISCUSIÓN}

En la tabla 1, 2, 3 y 4 se muestran los resultados de las propiedades fisicoquímicas de 10 bebidas de café geisha natural, 7 de café pacamara natural, 10 de café geisha lavado y 7 de café pacamara lavado, respectivamente. Las propiedades fisicoquímicas analizadas fueron: Acidez meq $100 \mathrm{~g}$, Brix \%, $\mathrm{pH}$, densidad $\mathrm{g} / \mathrm{mL}$, proteína \%, 5-CQA meq/100g, 4-CQA, meq/100g, 3-CQA meq/100g y Cafeína meq $/ 100 \mathrm{~g}$. También se representan las características del tostado dado por el color en Agtron y la humedad de la muestra de café verde usada para tostar, así como el puntaje dado por los jueces en la catación. Los resultados del análisis de regresión lineal múltiple se muestran en la tabla 5. Los parámetros que presentan valores $p$ menores son: 4-CQA y 3-CQA; por lo que se considera que estos son dos de los más significativos parámetros del modelo. Para futuras investigaciones se puede considerar calibrar modelos que consideren solamente los parámetros más significativos. El cuadrado del coeficiente de correlación ajustado es de $R_{\text {ajustado }}{ }^{2}=0.69$, lo cual indica una correlación media, y un error estándar de la estimación de 0.79 puntos. Estos son los primeros datos reportados del café variedad geisha y pacamara. Utilizando los coeficientes resultantes del modelo (redondeados a dos cifras significativas), se puede plantear el modelo mostrado en la ecuación (1). En este modelo, el 4-CQA, pH, AGTR, densidad y acidez tienen una influencia negativa sobre la calidad sensorial (CS), mientras que 3-CQA, 5CQA, humedad, Brix, y cafeína tiene una influencia positiva sobre CS. Con referencia al error estándar se puede esperar, en el $99.7 \%$ de los casos, que el valor de CS se encuentre en un rango de valores que inicia tres errores estándar por debajo del valor estimado y termina tres errores estándar por arriba del valor estimado, como se muestra en la ecuación (2)

$\mathrm{CS}_{\text {estimada }}=208-0.071 * 4 \mathrm{CQA}+0.0567{ }^{*} 3 \mathrm{CQA}-8 * \mathrm{pH}+0.0114$ * 5CQA -0.135 * AGTR -17.9 * proteína +

0.165 * humedad +0.754 * Brix -67.1 * densidad +0.000859 * cafeína -0.359 * acidez

$\mathrm{CS}_{\text {Rango-Estimado }}=\mathrm{CS}_{\text {estimada }} \pm 2.37$

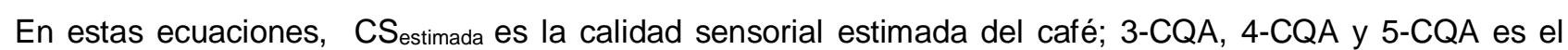
valor reportado en (meq/100g) del ácido 3-cafeoilquínico, 4-cafeoilquínico y 5-cafeoilquínico respectivamente de las bebidas de café; AGTR es el valor reportado para el grado de tostado del café

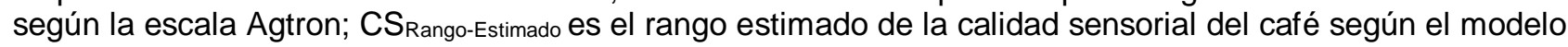
desarrollado.

El modelo presentado puede ser utilizado para estimar el rango en el cual se puede encontrar el puntaje del lote en consideración. Así, se espera que el modelo funcione como complemento para los caficultores que deseen evaluar su producto, si no tienen acceso a un grupo de jueces con cuya evaluación se pueda determinar un valor medio. En la tabla 6 se resumen los resultados de esta investigación y de 3 investigaciones de diferentes autores, donde se relaciona la calidad sensorial de muestras de café con parámetros fisicoquímicos. Si se analizan los datos de los otros estudios con el presentado aquí, se puede concluir que no se pueden comparar resultados que no tengan las mismas variables. Las variedades de café estudiadas en las 4 investigaciones son diferentes y fueron Geisha y Pacamara (en este estudio) y arábica y robusta (en los otros estudios); muestras de bebida de café,café verde y café tostado (en este estudio) y café verde, tostado y expreso (en los otros estudios); el tostado es diferente en cada caso; la instrumentación química para hacer análisis es variada, espectroscopía NIR, HPLC, química húmeda.

Barbosa et al., (2019), encontraron que algunos ácidos clorogénicos y la acidez bajan la calidad sensorial del café igual a lo encontrado en este estudio, pero el efecto de la cafeína es contrario en ambos estudios. Un modelo matemático para estimar la calidad de taza del café se debe construir controlando parámetros como variedad, tipo de tratamiento, grado de tostado, las propiedades fisicoquímicas, el tipo de instrumentación a utilizar, el tratamiento estadístico y puntuación de catación. Que hay que utilizar una gran cantidad de datos y validar el modelo. Por la importancia que tiene para Panamá la industria del café y que al mismo se le da un precio por la calidad de la taza obtenida en un proceso de cata, el modelo generado en este estudio se debe mejorar logrando un cuadrado del coeficiente de correlación ajustado $\left(R_{\text {ajustado }}{ }^{2}\right)$ mayor que el obtenido para que sea útil y apoye a la industria del café. Utilizando este modelo se podría estimar el puntaje de un café de manera rápida, barata y objetiva, complementando a la otorgada por los jueces en la catación. 


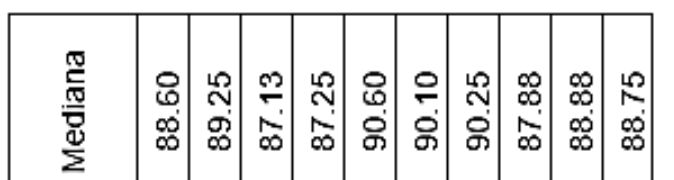

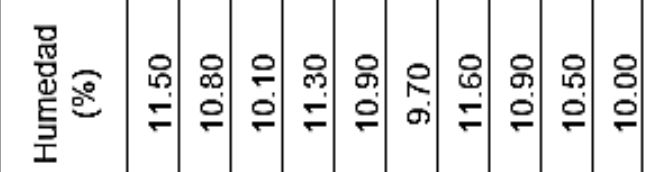

흥경

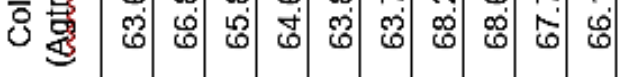

๘ี

岁照

迈号

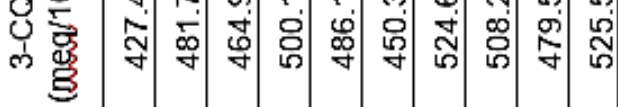

【ु号

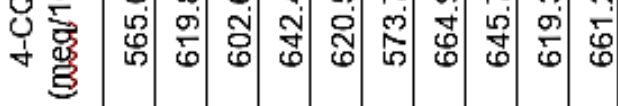

苛 ₹

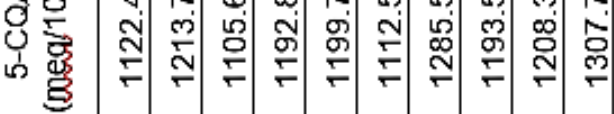

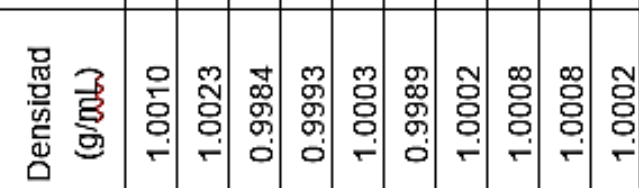

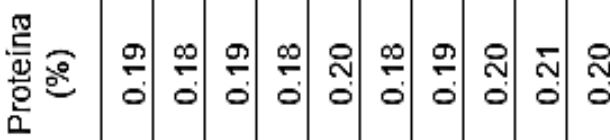

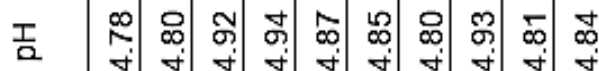

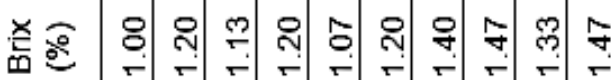

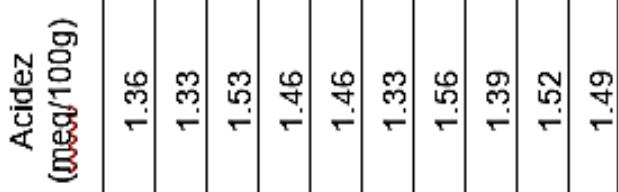

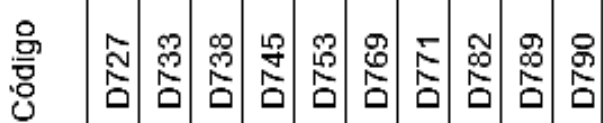

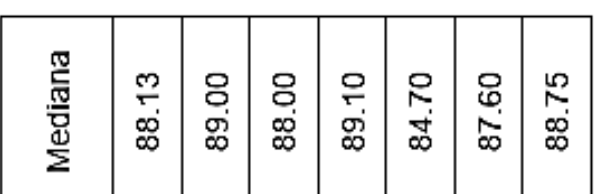

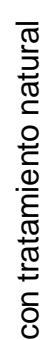

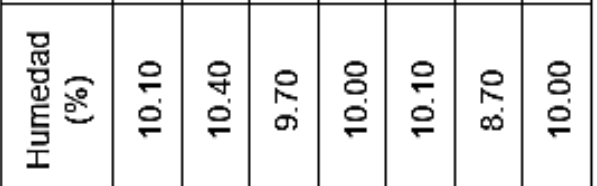

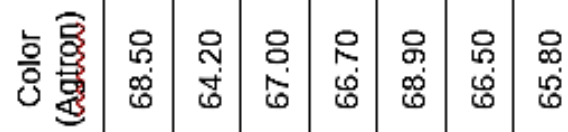

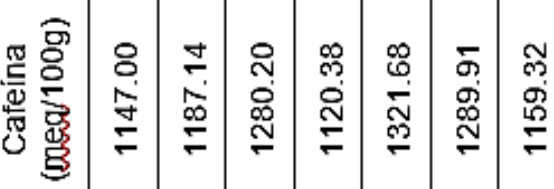

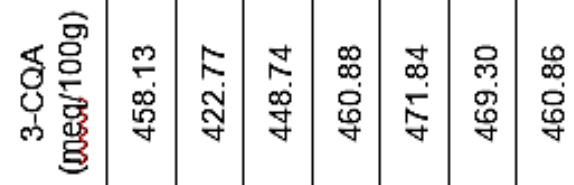

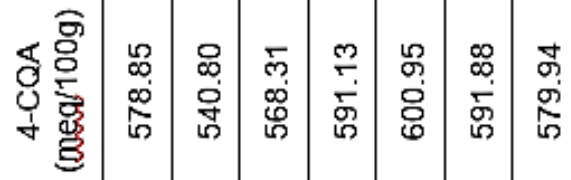

\begin{tabular}{|c|c|c|c|c|c|c|}
\hline 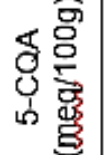 & 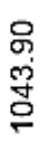 & $\begin{array}{l}\mathscr{\varrho} \\
\stackrel{\rho}{\sigma} \\
\kappa\end{array}$ & $\begin{array}{l}\text { N్ } \\
\text { ஸ్ }\end{array}$ & $\begin{array}{l}\text { J } \\
0 \\
\stackrel{0}{0} \\
\underline{0}\end{array}$ & 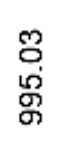 & 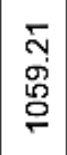 \\
\hline
\end{tabular}

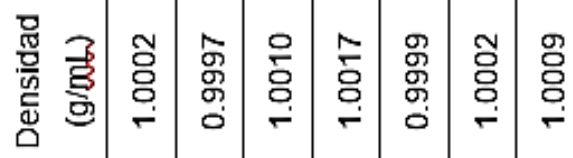

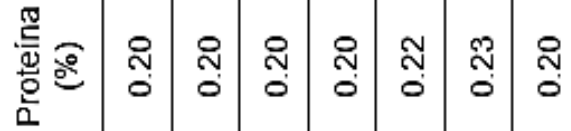

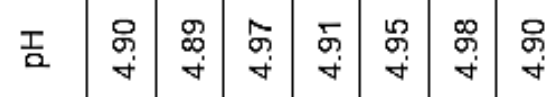

而き

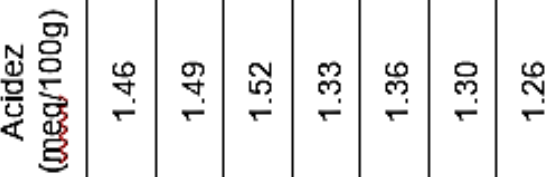

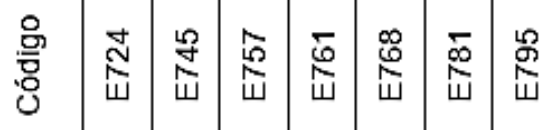




\begin{tabular}{|c|c|c|c|c|c|c|c|c|c|c|}
\hline 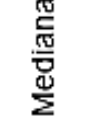 & $\begin{array}{l}\stackrel{L}{N} \\
\stackrel{\infty}{\infty}\end{array}$ & $\begin{array}{l}\stackrel{\text { N }}{8} \\
\text { g }\end{array}$ & \& & 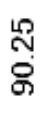 & $\begin{array}{l}\stackrel{9}{\circ} \\
\stackrel{\circ}{\circ}\end{array}$ & $\begin{array}{l}q \\
5 \\
\infty\end{array}$ & $\begin{array}{l}8 \\
\dot{\sigma}\end{array}$ & $\begin{array}{l}\text { 吕 } \\
\stackrel{\circ}{\infty}\end{array}$ & $\begin{array}{l}\stackrel{8}{\circ} \\
\infty \\
\infty\end{array}$ & $\begin{array}{l}\text { \&. } \\
\text { హู }\end{array}$ \\
\hline 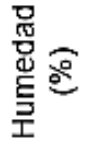 & 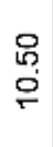 & 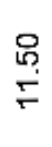 & 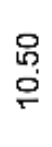 & 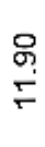 & $\begin{array}{l}\text { 号 } \\
\text { के }\end{array}$ & $\begin{array}{l}8 \\
\sigma \\
\sigma\end{array}$ & $\begin{array}{l}\text { Oे } \\
\text { ᄋ }\end{array}$ & 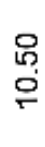 & $\begin{array}{l}\stackrel{8}{\infty} \\
\text { के }\end{array}$ & $\begin{array}{l}\text { 옴 } \\
\text { ᄋ }\end{array}$ \\
\hline 흥영 & $\begin{array}{l}8 \\
\stackrel{0}{0} \\
\stackrel{6}{6}\end{array}$ & 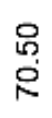 & $\begin{array}{l}\text { ถึ } \\
\text { هి }\end{array}$ & $\begin{array}{l}\text { 웅 } \\
\text { o }\end{array}$ & $\begin{array}{l}\text { ले } \\
\text { ळे }\end{array}$ & $\begin{array}{l}\text { సิ } \\
\text { ڤై }\end{array}$ & \begin{tabular}{l}
8 \\
\multirow{6}{0}{}
\end{tabular} & $\begin{array}{l}? \\
\text { i }\end{array}$ & & $\begin{array}{l}\circ \\
0 \\
6 \\
6\end{array}$ \\
\hline 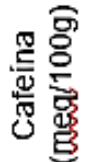 & 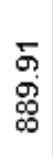 & $\begin{array}{l}\overline{6} \\
\stackrel{5}{\circ} \\
\stackrel{0}{0}\end{array}$ & 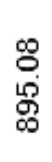 & 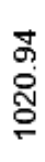 & 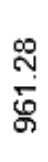 & 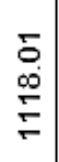 & $\begin{array}{l}0 \\
\infty \\
\stackrel{8}{\circ} \\
\infty \\
\infty\end{array}$ & $\begin{array}{l}\frac{\varphi}{\sigma} \\
\stackrel{\sigma}{\sigma}\end{array}$ & 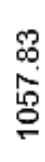 & $\begin{array}{l}\stackrel{R}{\circ} \\
\stackrel{\circ}{\circ}\end{array}$ \\
\hline
\end{tabular}

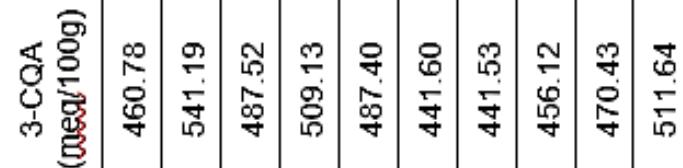

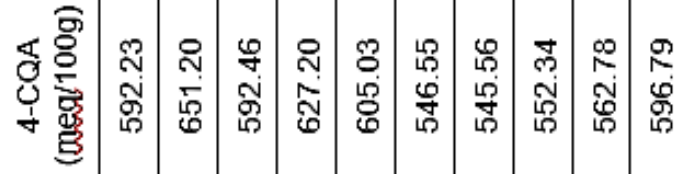

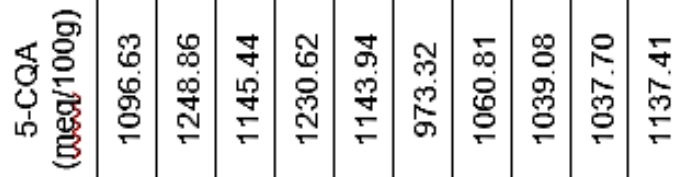

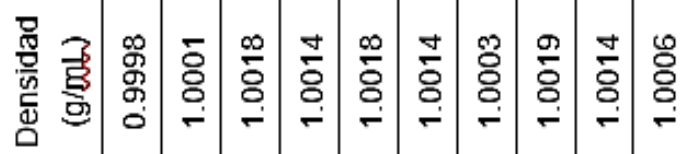

产

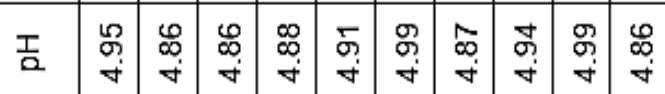

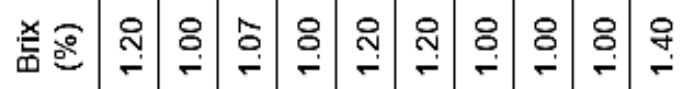

产旁

兽

\begin{tabular}{|c|c|c|c|c|c|c|c|}
\hline 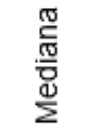 & 号 & $\begin{array}{l}\infty \\
\infty \\
\dot{\infty} \\
\infty\end{array}$ & $\begin{array}{l}\stackrel{\mu}{\rho} \\
\infty \\
\infty \\
\infty\end{array}$ & $\begin{array}{l}8 \\
\infty \\
\infty \\
\infty\end{array}$ & $\begin{array}{l}\infty \\
\infty \\
\infty \\
\infty\end{array}$ & $\underset{\infty}{\stackrel{\sim}{\infty}}$ & 宅 \\
\hline 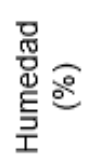 & $\begin{array}{l}\stackrel{0}{1} \\
\stackrel{0}{\circ}\end{array}$ & $\begin{array}{l}\stackrel{O}{\infty} \\
\stackrel{+}{\Gamma}\end{array}$ & 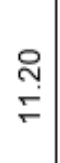 & $\begin{array}{l}\stackrel{O}{\circ} \\
\stackrel{+}{\Gamma}\end{array}$ & 음 & 울 & $\begin{array}{l}\circ \\
\stackrel{6}{\sigma} \\
\sigma\end{array}$ \\
\hline 흥 영 & $\begin{array}{l}\text { 우 } \\
\infty \\
0\end{array}$ & 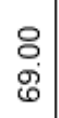 & $\begin{array}{l}\stackrel{2}{2} \\
\stackrel{0}{0}\end{array}$ & $\begin{array}{l}0 \\
\hat{n} \\
\hat{\omega}\end{array}$ & 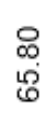 & $\begin{array}{l}\text { Оे } \\
00 \\
6\end{array}$ & 尺̃ \\
\hline
\end{tabular}

ชิ

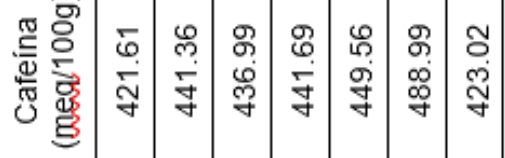

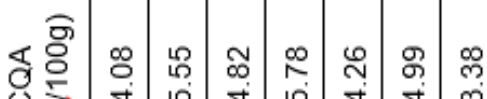

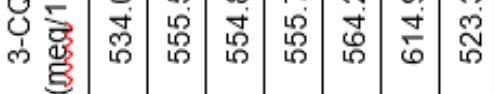

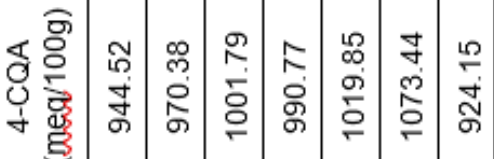

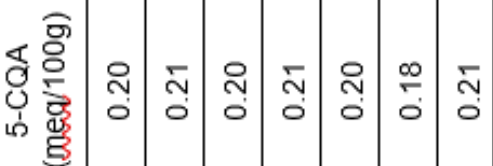

栗

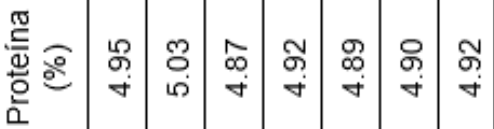

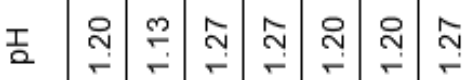

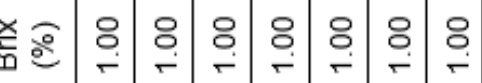

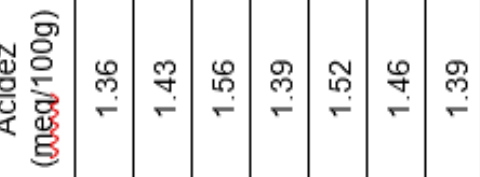

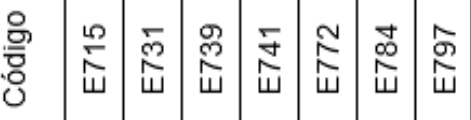


Tabla 5: Resultados de la regresión lineal utilizando 11 parámetros físico-químicos

\begin{tabular}{|l|c|c|c|}
\hline \multicolumn{4}{|c|}{ Estadísticas de la regresión } \\
\hline $\mathrm{R}_{\text {ajustado }}{ }^{2}$ & 0.69 & Error estándar & 0.79 \\
\hline Parámetros & Coeficientes & Error estándar & Valor-p \\
\hline Intercepto & 207.6834 & 201.6089 & 0.3141 \\
\hline 4-CQA & -8.1139 & 3.9011 & 0.0014 \\
\hline 3-CQA & 0.0114 & 0.0065 & 0.0015 \\
\hline Ph & -0.0710 & 0.0194 & 0.0494 \\
\hline 5-CQA & 0.0567 & 0.0156 & 0.0918 \\
\hline Agtron & 0.1653 & 0.2185 & 0.1829 \\
\hline Proteína & -0.3592 & 2.2340 & 0.3485 \\
\hline Humedad & -67.0865 & 202.8296 & 0.4572 \\
\hline Brix & 0.7535 & 1.2493 & 0.5526 \\
\hline Densidad & 0.0009 & 0.0032 & 0.7440 \\
\hline Cafeína & -17.8937 & 18.6811 & 0.7890 \\
\hline Acidez titulable & -0.1347 & 0.0979 & 0.8737 \\
\hline
\end{tabular}

Tabla 6: Investigaciones realizadas que relacionan parámetros fisicoquímicos con la calidad sensorial de muestras de café

\begin{tabular}{|c|c|c|c|c|}
\hline $\begin{array}{l}\text { Investigaciones } \\
\text { realizadas }\end{array}$ & $\begin{array}{l}\text { Muestras } \\
\text { estudiadas }\end{array}$ & $\begin{array}{l}\text { Parámetros } \\
\text { estudiados }\end{array}$ & Técnica utilizada & Resultados \\
\hline $\begin{array}{l}\text { Vega et al. Sin } \\
\text { publicar }\end{array}$ & $\begin{array}{l}\text { Bebida de Café, } \\
\text { café verde y } \\
\text { tostado, variedad } \\
\text { Geisha y Pacamara }\end{array}$ & $\begin{array}{l}\text { Acidez, brix, pH, } \\
\text { proteína, densidad, 5- } \\
\text { CQA, 4-CQA, 3-CQA, } \\
\text { cafeína, color, } \\
\text { humedad y puntaje de } \\
\text { cata. }\end{array}$ & $\begin{array}{l}\text { Química húmeda } \\
\text { y HPLC }\end{array}$ & $\begin{array}{l}\text { 4-CQA, pH, AGTR y acidez bajan } \\
\text { la CT de la taza, 5-CQA, 3-CQA, } \\
\text { humedad, brix y cafeína suben la } \\
\text { CT de la taza. }\end{array}$ \\
\hline $\begin{array}{l}\text { Barbosa et al. } \\
(2019)\end{array}$ & Café arábica, verde & $\begin{array}{l}\text { Sacarosa, ácidos } \\
\text { clorogénicos totales, } \\
\text { proteína, cafeína, } \\
\text { lípidos, diterpenos y } \\
\text { puntaje de cata. }\end{array}$ & $\begin{array}{l}\text { Espectroscopia } \\
\text { infrarrojo cercano } \\
\text { (NIR) }\end{array}$ & $\begin{array}{l}\text { Cafeína, proteína, ácidos } \\
\text { clorogénicos y acidez bajan la CT } \\
\text { de la taza, sacarosa/acidez, } \\
\text { cafestol/kahweol suben la CT de } \\
\text { la taza. }\end{array}$ \\
\hline $\begin{array}{l}\text { Ribeiro et al. } \\
\text { (2011) }\end{array}$ & $\begin{array}{l}\text { Café arábica, } \\
\text { tostado }\end{array}$ & $\begin{array}{l}\text { Cafeína, trigonela, 5- } \\
\text { CQA, celulosa, lípidos, } \\
\text { sacarosa, caseína y } \\
\text { polisacáridos. }\end{array}$ & $\begin{array}{l}\text { Espectroscopía } \\
\text { infrarrojo cercano } \\
\text { (NIR) }\end{array}$ & $\begin{array}{l}\text { Lípidos y proteína relacionados al } \\
\text { atributo de cuerpo, cafeína y } \\
\text { ácidos clorogénicos al atributo } \\
\text { dulzura, ácidos clorogénicos a } \\
\text { acidez y sabor, Cafeína, trigonela, } \\
\text { ácidos clorogénicos, } \\
\text { polisacáridos, sacarosa y proteína } \\
\text { relacionados al atributo limpieza y } \\
\text { CT. }\end{array}$ \\
\hline $\begin{array}{l}\text { Farah et al. } \\
(2006)\end{array}$ & $\begin{array}{l}\text { Café arábica, verde } \\
\text { y tostado }\end{array}$ & $\begin{array}{l}\text { Sacarosa, cafeína, } \\
\text { trigonela, ácidos } \\
\text { clorogénicos. }\end{array}$ & HPLC & $\begin{array}{l}\text { Ácido clorogénico (3-4 } \\
\text { dicafeoilquinico) y cafeína } \\
\text { presentan alta correlación con la } \\
\text { calidad de la taza, 5-CQA } \\
\text { correlación con pobre calidad de } \\
\text { taza. }\end{array}$ \\
\hline
\end{tabular}

Como ilustración del posible uso del modelo, en la figura 1 y 2 se muestra una comparación de la estimación del modelo y las puntuaciones asignadas por los distintos jueces para cada uno de los 34 lotes utilizados, sirviendo el modelo como referencia para estimar el posible rango en que es más probable que se encuentre el puntaje de cada lote. No se debe considerar este ejemplo como verificación de la precisión del modelo, ya que se han estimado los valores de los mismos lotes utilizados para calibrarlo, sin embargo, se puede considerar como un ejemplo de su uso para evaluar lotes fuera de este grupo. 


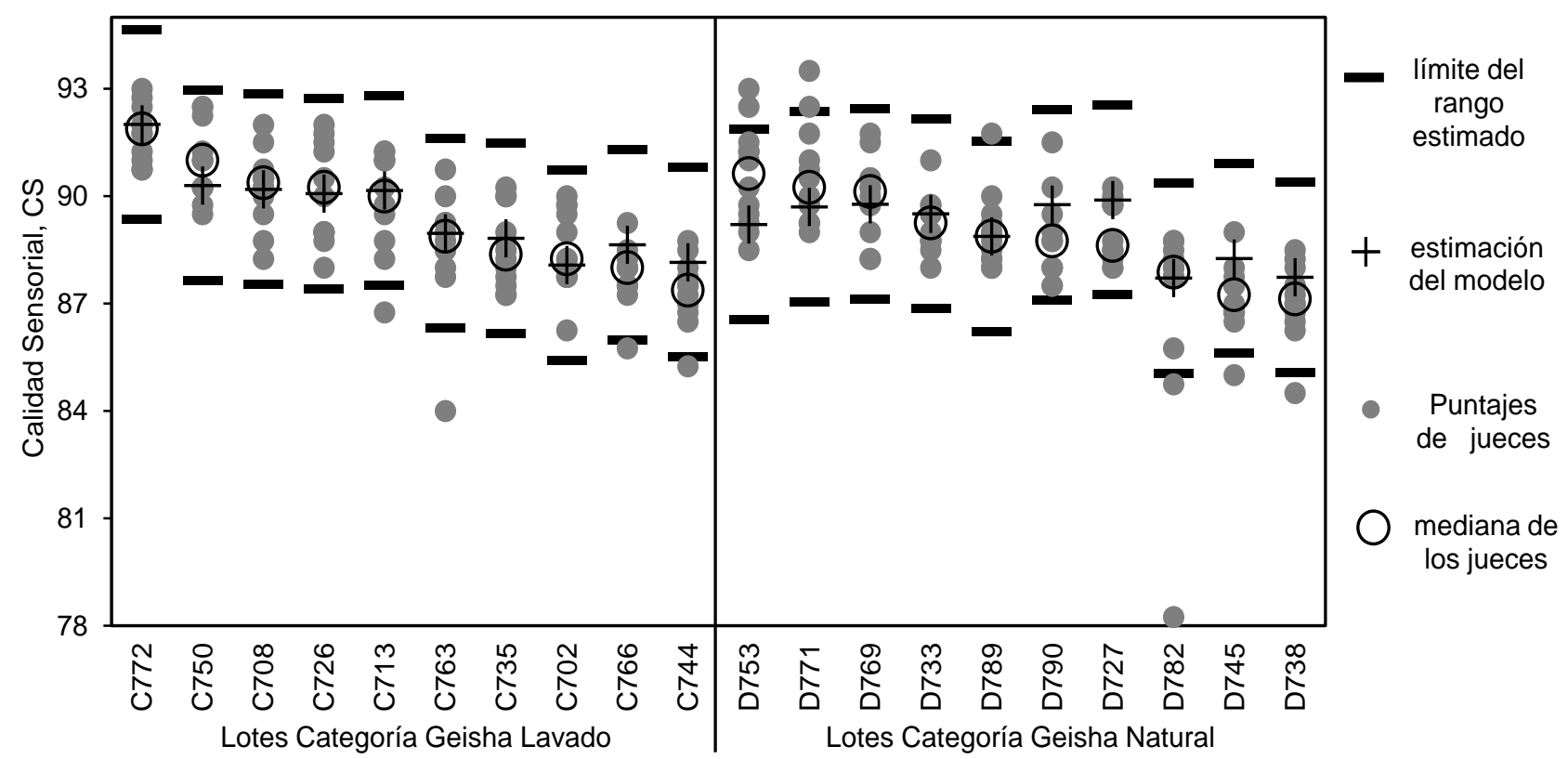

Fig. 1: Puntajes de 20 lotes de Geisha estudiados, mostrados por Categorías, incluyendo puntajes de los 12 jueces, Calidad Sensorial, CS (mediana de los puntajes de los jueces), valor estimado mediante el modelo, y rango de valores.

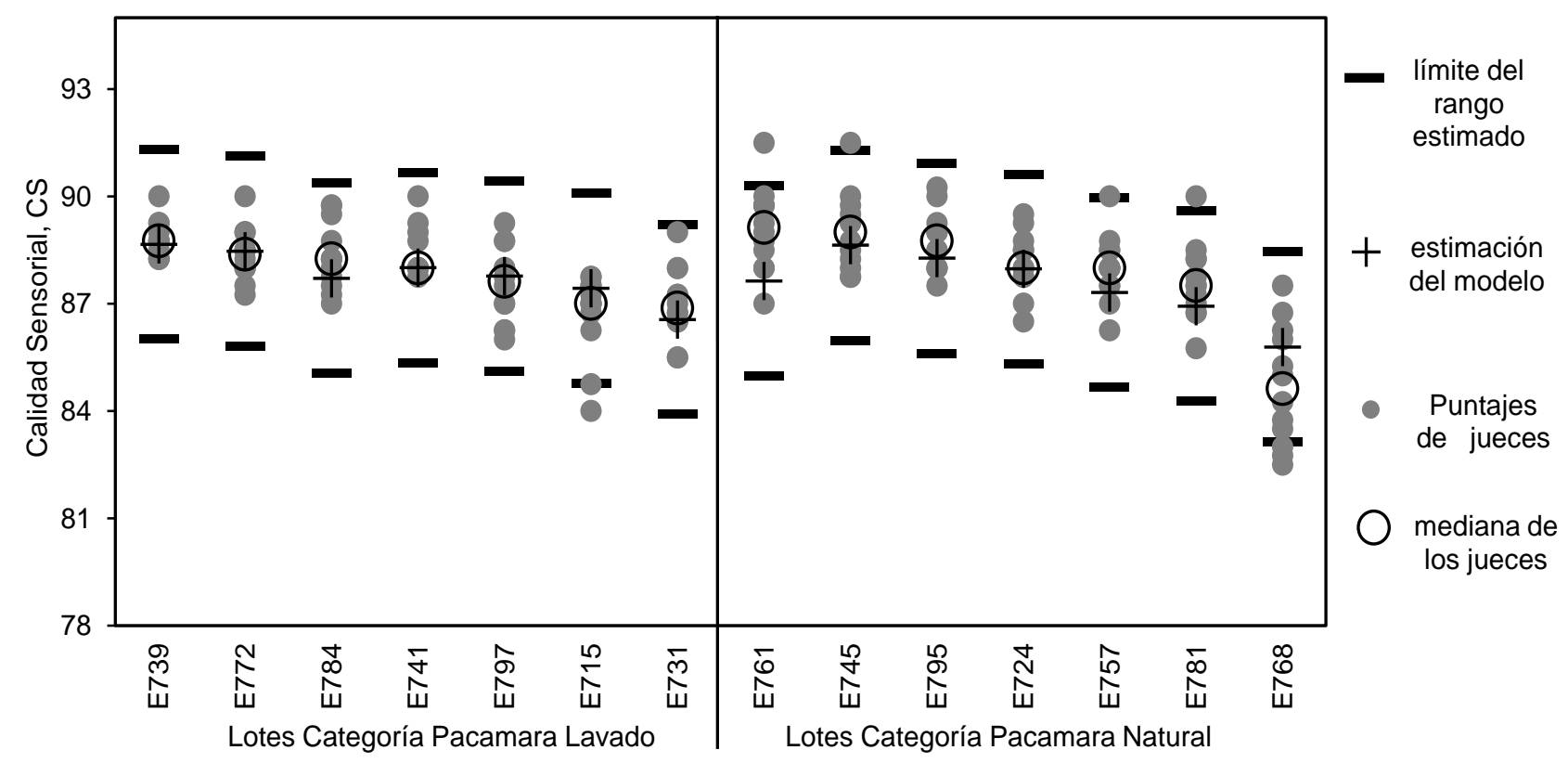

Fig. 2: Puntajes de 14 lotes de Pacamara estudiados por categorías, incluyendo puntajes de los 12 jueces, Calidad Sensorial, CS (mediana de los puntajes de los jueces), valor estimado mediante el modelo, y rango de valores.

En las figuras 1 y 2 se puede observar que CS se encuentra dentro del rango estimado por el modelo, así, cuando para un lote se encuentran puntajes fuera del rango estimado se puede recomendar revisar la puntuación asignada, ya que se estima que solo en 3 de cada 1000 casos $(0.3 \%)$ el valor de CS se encuentre fuera del rango estimado. Adicionalmente, se observa que la gran mayoría de los puntajes emitidos por los jueces se encuentran dentro de los límites superior e inferior del rango sugerido, en otras palabras, es atípico que los jueces asignen puntajes que caigan fuera del rango estimado mediante el modelo basado en los parámetros fisicoquímicos.

\section{CONCLUSIONES}

En este estudio se hizo un análisis de las propiedades fisicoquímicas y organolépticas de 34 muestras de café Geisha y Pacamara de Panamá. Con estos datos y utilizando la regresión lineal múltiple, se desarrolló un modelo matemático aceptable (con una correlación media, $\mathrm{R}_{\text {ajustado }}{ }^{2}=0.69$ ) para estimar la calidad sensorial del café (puntaje de cata) basado en 11 parámetros fisicoquímicos incluyendo propiedades del 
grano verde (\%humedad), del café tostado (color de tostado) y de la bebida del café. Los parámetros fisicoquímicos 4-CQA, 3-CQA y $\mathrm{pH}$, pueden ser considerados estadísticamente significativos para la estimación de la calidad sensorial del café, ya que se obtuvo valores p menores a $5 \%$, del análisis estadístico de la regresión. De estos parámetros, el 3-CQA presentó una correlación positiva, mientras que el 4-CQA y el pH presentaron una correlación negativa. El modelo presentado servirá para estimar el rango en el que se encuentra el puntaje de un determinado lote de café, y así puede ser utilizado como complemento por los caficultores que deseen evaluar la calidad de su producto.

\title{
NOTACIÓN
}

\author{
Abreviaciones \\ 5-CQA= ácido 5-cafeoilquínico \\ 4-CQA= ácido 4-cafeoilquínico \\ 3-CQA= ácido 3-cafeoilquínico \\ AGTR= escala de color Agtron \\ $\mathrm{CS}=$ calidad sensorial \\ $\mathrm{CT}=$ calidad total \\ CGAs= ácidos clorogénicos \\ Caf= cafeína \\ HPLC= cromatografía líquida de alta eficacia \\ $\mathrm{NIR}=$ Espectroscopia infrarrojo cercano
}

\section{AGRADECIMIENTOS}

Este estudio fue posible gracias al apoyo del Sistema Nacional de Investigación de la Secretaria Nacional de Ciencia Tecnología e Innovación de Panamá, a la Asociación de Cafés Especiales de Panamá, al Centro de Especialidades Agrícolas de café, S.A., a la Vicerrectoría de Investigación y Posgrado de la Universidad Autónoma de Chiriquí, al Instituto de Investigación Agropecuaria de Panamá y a la Fundación UNACHI.

\section{REFERENCIAS}

AOAC. Official Methods of Analysis of AOAC International,18th Ed., AOAC International, Maryland, USA (2010).

Barbosa, M. de S. G., Scholz, M. B. dos S., Kitzberger, C. S. G., y Benassi, M. de T., Correlation between the Composition of Green Arabica Coffee Beans and the Sensory Quality of Coffee Brews, doi.org/10.1016/j.foodchem.2019.04.072, Food Chemistry, 292, 275-280 (2019).

Bressanello, D., Liberto, E. y otros cinco autores. Coffee aroma: Chemometric Comparison of the Chemical Information Provided by Three Different Samplings Combined with GC-MS to describe the Sensory Properties in Cup, doi.org/10.1016/j.foodchem.2016.07.088, Food Chemistry, 214, 218-226 (2017).

Bustos-Vanegas, J. D., Bustos-vanegas, J. D., Cesar, P., y Arêdes, M., Developing Predictive Models for Determining Physical Properties of Coffee Beans During the Roasting Process Industrial Crops \& Products Developing Predictive Models for Determining Physical Properties ff Coffee Beans During the Roasting Process, doi.org/10.1016/j.indcrop.2017.12.015, Industrial Crops \& Products, 112, 839-845 (2017).

Caporaso, N., Whitworth, M. B., Grebby, S., y Fisk, I. D., Non-Destructive Analysis of Sucrose, Caffeine and Trigonelline on Single Green Coffee Beans by Hyperspectral Imaging, doi.org/10.1016/j.foodres.2017.12.031, Food Research International, 106, 193-203 (2018).

Cheng, B., Furtado, A., Smyth, H. E., y Henry, R. J., Influence of Genotype and Environment on Coffee Quality, doi.org/10.1016/j.tifs.2016.09.003, Trends in Food Science and Technology, 57, 20-30. (2016).

da Rosa, J., Freitas-Silva, O., de Oliveira, R., y de Rezende, C., Roasting Effects on Nutritional and Antinutritional Compounds in Coffee; in Food Processing Technologies, pp 61-90, CRC Press (2016).

Farah, A., Monteiro, M. C. y otros tres autores. Correlation Between Cup Quality and Chemical Attributes of Brazilian Coffee, doi.org/10.1016/j.foodchem.2005.07.032, Food Chemistry, 98(2), 373-380 (2006).

Laukalēja, I. y Krūma, Z., Quality of Specialty Coffee: Balance Between Aroma, Flavour an Biological Active Compound Composition: Review, Research for Rural Development, 240-247 (2018).

Naegele, E., Determination of Caffeine in Coffee Products According to DIN 20481, Agilent Technologies, Application note, 1-6 (2016). 
Pérez-Hernández, L. M., Chávez-Quiroz, K., Medina-Juárez, L. Á., y Gámez, N., Antioxidante de Café Verde y Procesado de las Especies Coffea Arabica y Coffea Canephora, Biotecnia, 15(1), 51-56 (2012).

Ribeiro, J. S., Ferreira, M. M. C., y Salva, T. J. G., Chemometric Models for the Quantitative Descriptive Sensory Analysis of Arabica Coffee Beverages Using Near Infrared Spectroscopy, doi.org/10.1016/j.talanta.2010.11.00, Talanta, 83(5), 1352-1358 (2011).

SCAA, Cupping Specialty Coffee, Specialty Coffee Asociation of America, pp 1-10, California, United States, (2015).

Schenker, S., y Rothgeb, T., The Roast-Creating the Beans' Signature, doi.org/10.1016/B978-0-12-803520-7.00011-6, The Craft and Science of Coffee, 245-271 (2017).

Toci, A. T., y Boldrin, M. V. Z., Coffee Beverages and Their Aroma Compounds, doi.org/10.1016/B978-0-12-8115183.00012-0, In Handbook of Food Bioengineering, Natural and Artificial Flavoring Agents and Food Dyes, pp. 397-425, Academic Press, (2018).

Tolessa, K., Rademaker, M., De, B., y Boeckx, P., Prediction of Specialty Coffee Cup Quality Based on Near Infrared Spectra of Green Beans, doi.org/10.1016/j.talanta.2015.12.039, Talanta, 74, 150-367 (2015).

Vega, A., Reyes, S. y otros tres autores. Cuantificación de Cafeína en Cafés Comerciales de Panamá, Ciencia y Tecnología, 30(2), 57-64 (2014).

Vega, A., De León, J. y Reyes, S.M., Determinación del Contenido de Polifenoles Totales, Flavonoides y Actividad Antioxidante de 34 Cafés Comerciales de Panamá, Información Tecnológica, 28(4), 29-38 (2017).

Vega, A., De León, J., Reyes, S.M. y Miranda, S.Y., Componentes Bioactivos de Diferentes Marcas de Café Comerciales de Panamá. Relación entre Ácidos Clorogénicos y Cafeína, Información Tecnológica, 29(4), 43-54 (2018).

Wei, F., Furihata, K., Miyakawa, T., y Tanokura, M., A Pilot Study of NMR-Based Sensory Prediction of Roasted Coffee Bean Extracts, doi.org/10.1016/j.foodchem.2013.11.161, Food Chemistry 152, 363-369 (2014).

Wen, D., Liu, Y., Liao, Y., y Liu, H., A Universal HPLC Method for the Determination of Phenolic Acids in Compound Herbal Medicines a Universal HPLC Method for the Determination of Phenolic Acids, J. Agric. Food Chem., 53(17), 6624-6629 (2005). 
